\title{
Experimental investigation of noise characteristics for HVAC silencers
}

\author{
Carmen Bujoreanu*, and Marcelin Benchea \\ Technical University “Gheorghe Asachi” Iaşi, Mechanical Engineering Faculty, Department of \\ Mechanical Engineering, Mechatronic\&Robotics, 700050 Iaşi, Romania
}

\begin{abstract}
Heating, ventilating and air-conditioning (HVAC) systems represent a major source of inside buildings noise with a negative effect on the acoustical environment. Therefore, it is necessary to add attenuation to the system. This is provided by sound attenuators or silencers manufactured in different geometrical configurations and with various absorptive lining materials. The paper deals with the insertion loss, as a performance criterion for in-duct noise, of some dissipative silencers types. The measured data are compared in order to establish the efficiency of every tested silencer type. Third-octave band analysis of random noise of an air handling unit (AHU) from a HVAC system is realized without and with silencers (ducts fitted). Insertion loss measurements are made in an anechoic room using a test facility that agrees the requirements of ISO 7235:2009. The results highlight the effects of the silencers geometry, pressure-air flow and loading relations upon the sound attenuation level. The silencers selection could be a difficult task for the designers which have to consider all these parameters.
\end{abstract}

\section{Introduction}

Silencers, also called sound attenuators, absorbers or mufflers, are designed to reduce the noise transmitted from a source to a receiver. In the HVAC field, the noise attenuation in ventilation ducts becomes of major interest as the acoustic comfort requirements have become more stringent. The European Standards EN ISO 7235:2009 (EN ISO 2009a) and EN ISO 11691:2009 (EN ISO 2009b) and the American Standard ASTM E477-06a (ASTM 2006) set the experimental measurement procedures in order to determine the insertion loss (attenuation), in frequency bands, of ducted silencers and air-terminal units with and without airflow.

For HVAC applications, the most common silencers are duct silencers, installed on the intake and/or discharge side of a fan or air handler. Also, they may be used on the receiver side of other noise generators such as terminal boxes, valves, dampers, etc. There are several types of silencers used in the sound attenuation: reactive, active and dissipative [1].

For the wide frequency bands, the dissipative silencers are recommended. They attenuate the sound especially through the presence of the sound-absorbing materials with a certain

\footnotetext{
*Corresponding author: carmen.bujoreanu@gmaill.com
} 
flow resistivity. These silencers are the most widely used for noise control where fan noise, flow induced noise and engine noise must be attenuated [2].

The duct silencers are available in various shapes, including rectangular, round, elbow ones, and different sizes in accord to the duct design. Usually, a commercial duct silencer's outer appearance is similar to a piece of duct. It consists of a sheet metal casing with length commonly ranging from $(1-3 \mathrm{~m})[2]$. Sound-absorbing material lining one or more of the silencer sides, in a usual configuration. Other silencer's configurations include a centrally located bar or several splitters mounted horizontally or vertically inside it [2].

Some comparisons with experimental data revealed noticeable difference between practice and theory and emphasize the limitations of theoretical approaches for the silencer design $[3,4,5]$.

The paper is an experimental work and it deals with the insertion loss, as a performance criterion for in-duct noise, of some dissipative silencers types, intended to be commercialized, from the same producer. The measured data are compared in order to establish the efficiency of every tested silencer type. Third-octave band analysis of random noise of an air handling unit (AHU) from a HVAC system is realized without and with silencers (ducts fitted). Insertion loss measurements are made using the test facility that agrees the requirements of ISO 7235:2009 [6]. The results highlight the effects of the silencers geometry, pressure-air flow relation and loading upon the sound attenuation level and lead to a better silencer design.

\section{Experimental methodology}

The lined silencers used in specific HVAC systems need to consider some parameters as: the air-flow direction and velocity, the pressure drop after the silencer insertion and the most important, the insertion loss in decibels $(\mathrm{dB})$ in each octave band. The use of silencer naturally produces the fan loading increasing and both the acoustic and air flow performances must be evaluated when they are designed $[7,8]$.

ISO 7235: 2009 specifies the methods for determining the insertion loss, in frequency bands, of ducted silencers with and without airflow, the sound power level of the flow noise generated by ducted silencers, the total pressure loss of silencers with airflow, and the transmission loss of air-terminal units.

The test facility for measurements of insertion loss in dynamic conditions, with airflow, is presented below.

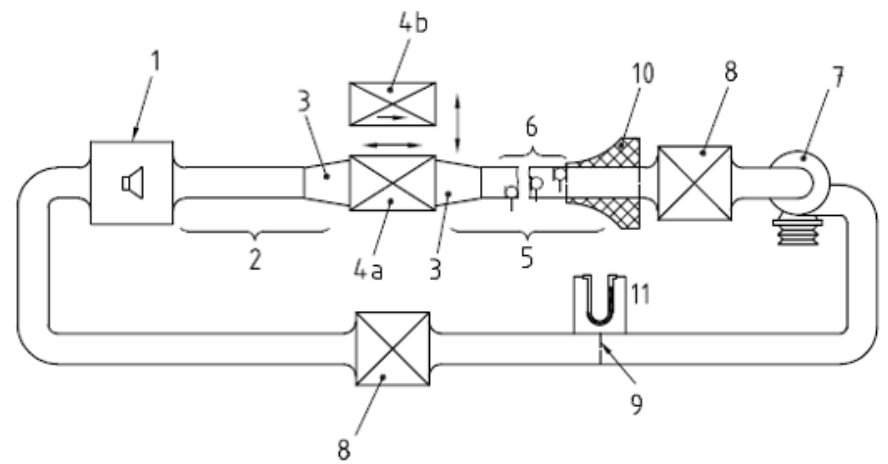

Fig. 1. Test facility for measurements of insertion loss with airflow and/or flow noise Arrangement for in-duct measurements (ISO 7235: 2009).

The elements of the test facility presented in Fig. 1 are: 1 - loudspeaker unit in the source chamber ; 2 - test duct in front of the test object ; 3 - transitions ; $4 \mathrm{a}$ - test object ; $4 \mathrm{~b}$ - 
substitution duct ; 5 - test duct behind the test object ; 6 - wind-shielded microphones ; 7 fan (installed to generate either forward or reverse flow relative to the direction of sound propagation ; 8 - fan silencers ; 9 - nozzle for measurement of flow rate (alternatives: orifice or Venturi nozzle) ; 10 - anechoic termination (we have used an anechoic room) ; 11- static pressure measurement

The experimental setup for our measurements consists of: centrifugal fan providing a variable airflow (from $1 \mathrm{~V}$ to $10 \mathrm{~V}$ with a ratio of $1 \mathrm{~V}$ ), device for measuring the flow rate (anemometer), device for measuring the pressure drop (pressure differential meter), special sound-source equipment (consists of a white noise generator B\&K, an amplifier and a broad band loudspeaker), the test object (the dissipative silencers), the transition elements on either side of the test object, special receiving-sound equipment (soundmeter B\&K connected with the microphone and NIDAQ board, laptop with LabVIEW soft compatible with National Instruments DAQPad for data processing).

The insertion loss $D_{I}$ of the test object represents the reduction in the sound power level in the duct behind the test object, due to the insertion of the test object into the duct in place of a substitution duct. It is given by the equation:

$$
D_{I}=S W L_{I I}-S W L_{I}[\mathrm{~dB}]
$$

where: $S W L_{I}$ - the level of the sound power in the frequency band considered, propagating along the test duct when the test object is installed; $S W L_{I I}$ - the level of the sound power in the frequency band considered, propagating along the test duct when the substitution duct replaces the test object.

The insertion loss, $D_{I}$, must be measured from spatially energy-averaged sound pressure levels $S P L$ at identical points or paths. In one test series, $S P L_{I}$ is determined with the test object installed and in a further series, $S P L_{I I}$ is determined with the test object replaced by the substitution duct [6].

The insertion loss measurements were realized according to the procedure for different airflows variations of the centrifugal fan (from $1 \mathrm{~V}$ to $10 \mathrm{~V}$ with a ratio of $1 \mathrm{~V}$ ) and the white noise generated from sound-source equipment.

The experimental setup and the tested silencers are presented in Fig.2.
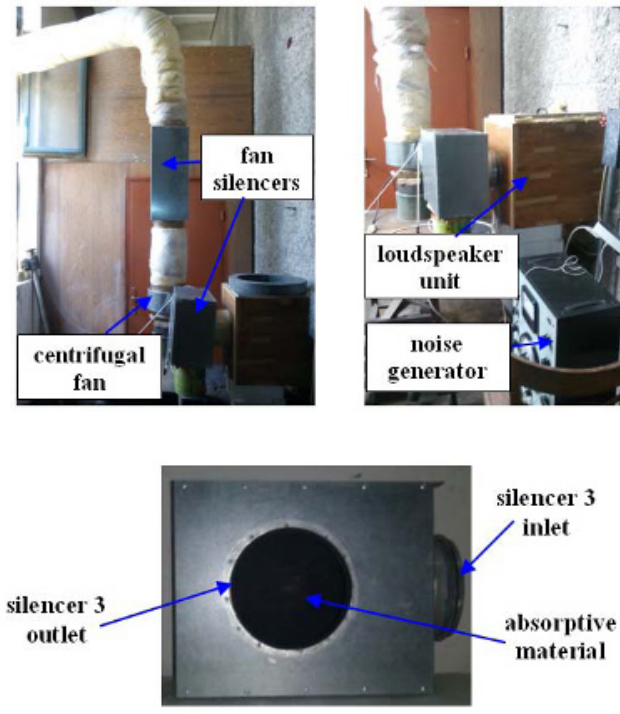
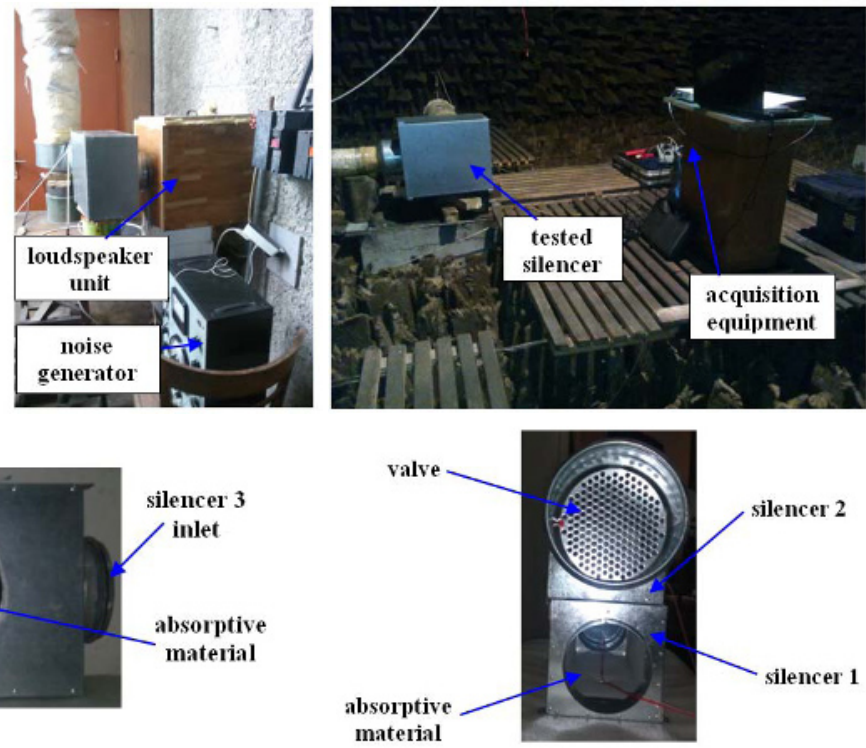

Fig. 2. Experimental setup outside and inside the anechoic room. 
The tested silencers, with different configurations, are noted with:

- "silencer 1"- the straight silencer with valve $(\Phi 125 / \Phi 125 \mathrm{~mm}$ and inlet-outlet air flow direction is $\left.180^{\circ}\right)$; the valve can be open or closed.

- "silencer 2" - the elbow silencer with valve (Ф125/Ф125 mm and inlet-outlet air flow direction is $90^{\circ}$ rotated); the valve can be open or closed.

- "silencer 3" - the straight silencer without valve $(\Phi 125 / \Phi 125 \mathrm{~mm}$ and inlet-outlet air flow direction is $90^{\circ}$ rotated).

The absorptive material lining the silencers 1 and 2 is different from that lining the silencer 3, so their acoustical properties, in terms of sound absorption, are not the same.

First of all, we have measured the noise level $\mathrm{dB}(\mathrm{A})$, A weighted, by means the LabVIEW soft, using the third octave analysis of the sound pressure levels, $\mathrm{dB}(\mathrm{A})$, for nominal frequencies of $63,125,250,500,1000,2000,4000,8000 \mathrm{~Hz}$ and total band power (within any specified frequency band). Then, we have calculated, according to relation (1), the insertion loss for every silencer tested.

\section{Results and discussions}

We have selected some experimental results to be presented, comparatively, in the following figures. Figure 3 reports the pressure drop versus the air flow and Figs. 4 and 5 express the sound attenuation (in terms of sound pressure) in the specified silencers. It should be noted that, according to the manufacturer, the most used regime in HVAC exploitation corresponds to 4-6 V loading. The sound attenuation for this loading is of interest. However, we also present some results acquired in the extreme regime of $10 \mathrm{~V}$.

Figure 3 compares the pressure-air flow evolution for the specified silencers of the same inlet-outlet dimensions, $\Phi 125 / \Phi 125$, but different air flow direction, during the most unfavorable loading range $(10 \mathrm{~V})$.

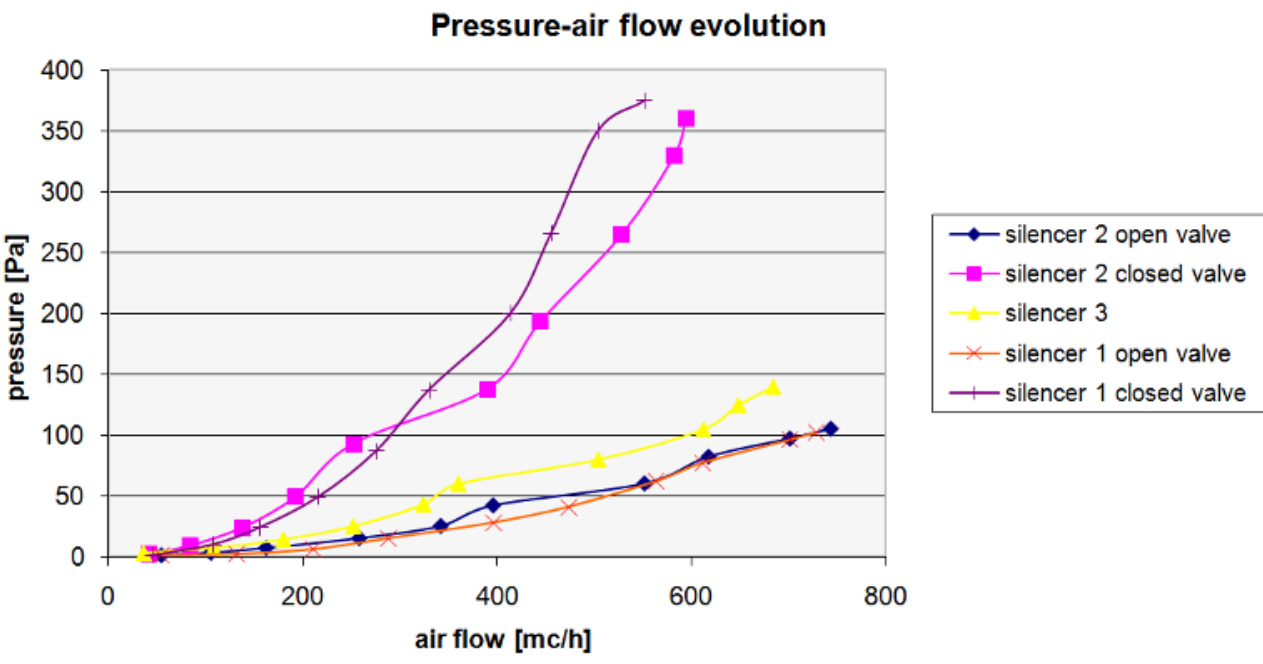

Fig. 3. Pressure versus air flow.

Note that we record the same evolution using silencer 1 open valve as for silencer 2 open valve, even the air flow direction is $90^{\circ}$ rotated. Instead, for approximately the same pressures, the air flow values are greater for silencer 2 closed valve than those for silencer 1 closed valve, therefore the rotated direction of air flow is important in the closed valve configuration. Obviously, there are noticeable differences between open and closed variants, in terms of pressure. Also, the loading increasing leads to the increase of the pressure-air flow 
curve slope. There are little differences between the silencer 3 (another absorptive material used inside) and silencer 1 open valve values on the pressure-air flow curve, so the lining has no influence on these parameters evolution, as it was expected.

Figures 4 and 5 report the sound attenuation versus the octave band frequencies, for the loading of interest in HVAC exploitation. We compared the sound attenuation for the three silencers types with the same inlet-outlet, but different flow direction and lining. As a general observation, we emphasize the attenuation decreasing with the loading increasing for all silencers analyzed.

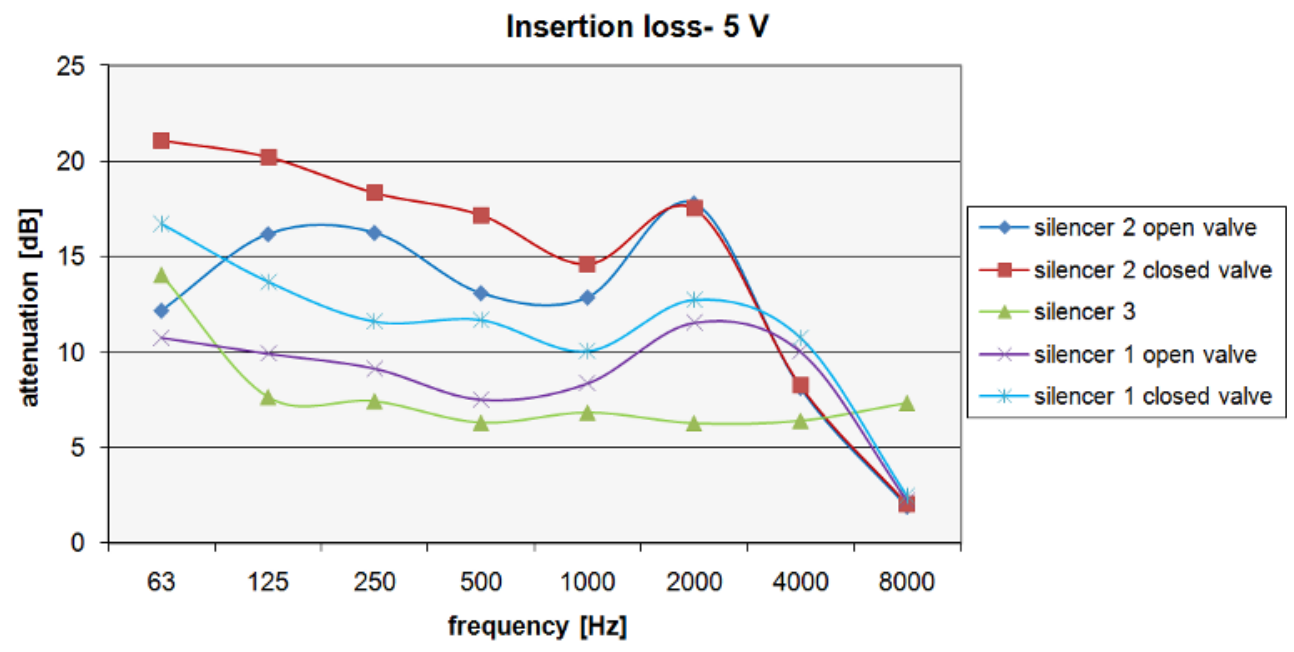

Fig. 4. Insertion loss for $5 \mathrm{~V}$ loading.

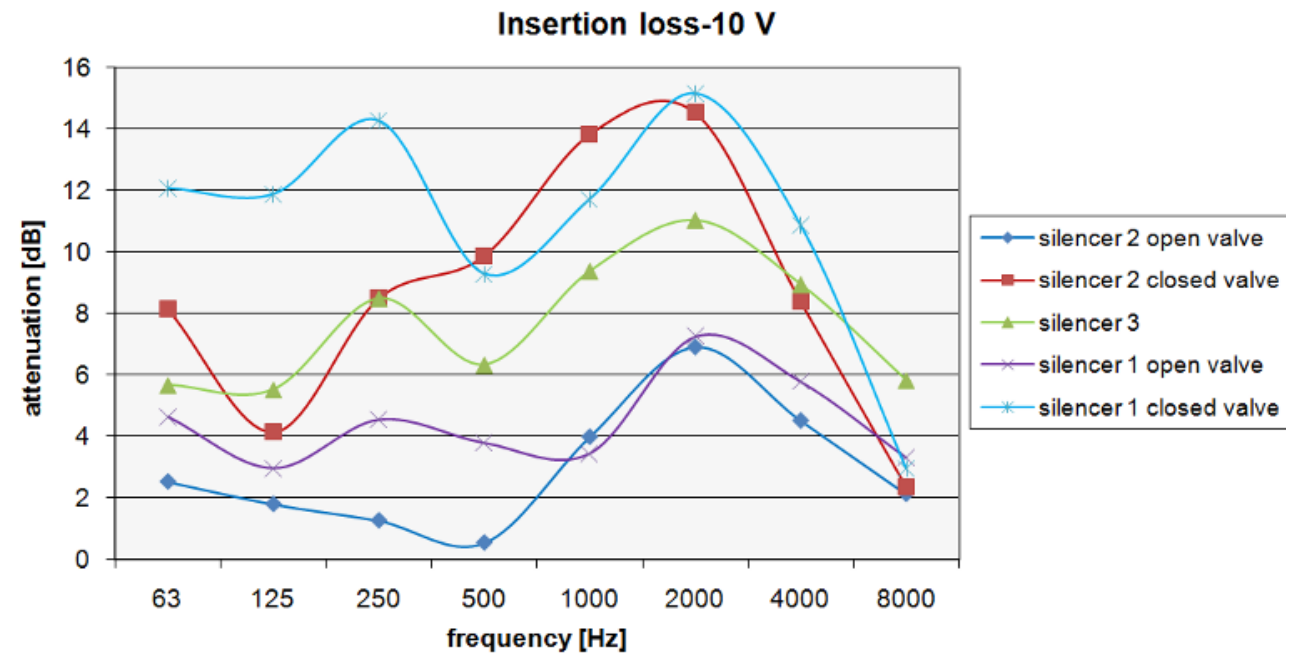

Fig. 5. Insertion loss for $10 \mathrm{~V}$ loading.

With the loading increasing, at $5 \mathrm{~V}$ (Fig. 4) - considered the usual exploitation of HVAC - we remark the good attenuation at lower frequencies, under $125 \mathrm{~Hz}$, and a poor one above $4000 \mathrm{~Hz}$. Silencer 2 ensure the best sound attenuation from the analyzed types and we already observed the closed valve advantage in this case. The attenuation values decrease by 5-6 $\mathrm{dB}$ 
in $250-500 \mathrm{~Hz}$ frequencies area, compared with those from $1 \mathrm{~V}$ loading, but they are growing with 5-6 dB for lower frequencies under $250 \mathrm{~Hz}$. Silencer 3 (different absorptive lining) offers a weaker attenuation in the middle frequencies, compared to the other discussed silencers. However, we observe that at low (under $250 \mathrm{~Hz}$ ) and high frequencies (over $4000 \mathrm{~Hz}$ ), silencer 3 has a good sound attenuation behaviour.

Figure 5 presents the extreme situation, less used by potentially beneficiaries, when the loading reaches its maximum $(10 \mathrm{~V})$. The best sound attenuation behaviour $(14-15 \mathrm{~dB})$ belongs to the silencer 1 closed valve, and the second place is occupied by the silencer 2 closed valve. We remark the silencer 3 for its satisfactory sound attenuation at $8000 \mathrm{~Hz}$ and certain constancy in attenuation behaviour along all the loading stages.

We can summarize all the results above presented for the tested silencers:

- silencer 2 (both open and closed valve configurations) provides a good attenuation in the middle frequencies range at every loading stage.

- silencer 3 offers a better attenuation in low and high frequencies and a moderate one in the middle frequencies range at every loading stage.

- silencer 1 closed valve is effective in sound attenuation at the maximum loading.

\section{Conclusions}

The paper is an experimental investigation on sound attenuation performances of some silencers types used for HVAC systems. The measurements were realized according to the standard and a test facility was implemented in an anechoic room. The results have emphasized the attenuation decreasing trend with the loading increasing of the tested silencers, as well as the important influence in attenuation of the absorptive material lining. The silencer lining influences the sound attenuation more than the geometry in certain situations. The loading increasing leads to the increase of the pressure-air flow curve slope. Also, the flow direction influences the acoustic level and improves the attenuation $\left(90^{\circ}\right.$ rotation of flow direction, as in silencer 2). The inlet-outlet size of the silencer, the length, and the acoustical properties of the lining must be well correlated, in accord to the purpose and needs to be covered through its use in a specific application. It's a difficult task for the silencer designer. Future work may concern a theoretical investigation on sound attenuation in a silencer, with precautions and knowledge gained through this experimental investigation.

\section{References}

1. ASHRAE, Handbook-Sound and Vibration Control 48, 279 (2011)

2. D. Borelli, C. Schenone, I. Pittaluga, Proc. of Meetings on Acoustics 14, 040004 (2012)

3. K.S. Peat, J. Sound Vibration 146, 353-360 (1991)

4. M. L. Munjal, B. K. Behera, P. T Thawani, Appl. Acoust. 54, 229-238 (1998)

5. T. W. Wu, C. Y. R Cheng, P. Zhang, J. Acoust. Soc. Am. 111, 2566-2572 (2002)

6. EN ISO $7235: 2009$, Acoustics-Measurement procedures for ducted silencers-Insertion loss, flow noise and total pressure (2009)

7. A. Bhatia, PDHOnline 1-72 (2012)

8. W.T.W Cory, Fans \& Ventilation: A Practical Guide (Elsevier, 424, 2005) 